\title{
A 50 años de la descripción del síndrome de insuficiencia respiratoria aguda
}

\author{
Raúl Carrillo-Esper, ${ }^{1}$ Gilberto Felipe Vázquez-De Anda, ${ }^{2}$ Cynthia Ivonne Mejía-Pérez, ${ }^{2}$ \\ María Guadalupe Delaye-Aguilar, ${ }^{3}$ Ana Ivonne Pérez-Castañeda, ${ }^{3}$ Jesús Carlos Briones-Garduño ${ }^{4}$ y \\ Manuel Antonio Díaz de León-Ponce ${ }^{1}$ \\ ${ }^{1}$ Academia Nacional de Medicina de México; ${ }^{2}$ Instituto Mexicano del Seguro Social, Centro Médico Nacional Siglo XXI, Hospital de Especialidades; \\ ${ }^{3}$ Centro de Investigación en Ciencias Médicas, Universidad Autónoma del Estado de México; ${ }^{4}$ Secretaría de Salud, Hospital General de México, \\ Servicio de Ginecología y Obstetricia. Ciudad de México, México
}

\section{Resumen}

En 1967, Ashbaugh et al. publicaron en Lancet la descripción de una nueva entidad para la que acuñaron el nombre "síndrome de distress respiratorio del adulto". En ese artículo describieron minuciosamente a 12 enfermos que presentaban insuficiencia respiratoria, con infiltración pulmonar bilateral e hipoxemia resistente a oxigenoterapia. Para su manejo se hizo énfasis en la importancia de la intubación y la ventilación mecánica con presión positiva al final de la espiración. A 50 años de la publicación se han logrado grandes avances en el conocimiento de esta enfermedad, lo que ha influido en el manejo y supervivencia de los pacientes. Para celebrar este cincuentenario, la Academia Nacional de Medicina de México organizó un simposio que tuvo como objetivos difundir el conocimiento de esta enfermedad, reconocer a los personajes que hicieron la descripción original y a quienes en 50 años de historia han contribuido a su mejor entendimiento. El simposio abordó los temas de interacción pulmón-riñón, bases moleculares de la enfermedad y avances en el tratamiento.

PALABRAS CLAVE: Síndrome de insuficiencia respiratoria aguda. Ventilación mecánica. Hipoxemia. Presión positiva al final de la espiración.

\begin{abstract}
In 1967, Ashbaugh et al. published in the Lancet the description of a new entity, for which they coined the name "adult respiratory distress syndrome". On that article, they thoroughly described 12 patients who had respiratory distress with bilateral pulmonary infiltrates and oxygen therapy-refractory hypoxemia. For its management, emphasis was made on the importance of intubation and mechanical ventilation with positive end-expiratory pressure. At 50 years of its first publication, great advances on the knowledge of this condition have been achieved, which has influenced on patient management and survival. To celebrate this $50^{\text {th }}$ anniversary, the National Academy of Medicine of Mexico organized a symposium with the purpose to spread the knowledge about this condition, recognize the researchers who made the original description and those who over the course of 50 years of history have contributed to its better understanding. The symposium addressed the topics of lung-kidney interaction, molecular bases of the disease and therapeutic advances.
\end{abstract}

KEY WORDS: Acute respiratory distress syndrome. Mechanical ventilation. Hypoxemia. Positive end-expiratory pressure.

Correspondencia:

Raúl Carrillo-Esper

E-mail: revistacma@ comexane.org
Fecha de recepción: 30-11-2017

Fecha de aceptación: 16-01-2018

DOI://dx.doi.org/10.24875/GMM.M18000062
Gac Med Mex. 2018;154:236-253

Disponible en PubMed www.gacetamedicademexico.com 


\title{
El síndrome de insuficiencia respiratoria aguda
}

\author{
Raúl Carrillo-Esper
}

\section{Introducción}

El 12 de agosto de 1967 apareció en la prestigiosa revista Lancet un artículo titulado "Acute Respiratory Distress in Adults", firmado por los doctores David G. Ashbaugh, Boyd Bigelow, Thomas L. Petty y Bernard Levine. El doctor Thomas L. Petty, distinguido neumólogo, fue pieza clave en la publicación. En ese trabajo, los autores describieron una entidad que previamente estaba mal conceptualizada, la definieron y establecieron los criterios diagnósticos y las bases del tratamiento; en pocas palabras, describieron una nueva enfermedad a la que denominaron de manera genérica "síndrome de dificultad respiratoria aguda en adultos" (SDRA o RDSA, respiratory-distress syndrome in adults) y la diferenciaron claramente del edema pulmonar secundario a insuficiencia cardiaca; en la actualidad, esta entidad se denomina síndrome de insuficiencia respiratoria aguda (SIRA) ${ }^{1}$ (Figuras 1 y 2 ).

La descripción de SDRA se fundamentó en la descripción de una serie de 12 enfermos que presentaban insuficiencia respiratoria aguda, cianosis resistente a tratamiento con oxígeno, disminución de la distensibilidad pulmonar e infiltrados difusos en la radiografía de tórax. El sustrato histopatológico que describieron era semejante al observado en pulmones de niños con insuficiencia respiratoria, a las atelectasias congestivas y a la lesión pulmonar posreperfusión. Microscópicamente se observó hemorragia, congestión, microatelectasias, daño alveolar difuso y membranas hialinas La mortalidad fue de $58 \%$, con una mejor oportunidad de supervivencia en los pacientes manejados con ventilación mecánica y presión positiva al final de la espiración (PPFE). ${ }^{1}$

A 50 años de la descripción de esta entidad, el panorama ha cambiado radicalmente: los avances en el conocimiento de sus bases genéticas, moleculares y fisiopatológicas han permitido un mejor entendimiento del comportamiento clínico de la enfermedad, lo que ha permitido el desarrollo de estrategias terapéuticas ventilatorias y no ventilatorias fundamentales para mejorar la supervivencia.

\section{Epidemiología}

El SIRA es una entidad compleja y heterogénea. Se considera un problema de salud pública debido a su

\author{
ACUTE RESPIRATORY DISTRESS \\ IN ADULTS \\ David G. Ashbaugh \\ M.D. Ohio State \\ ASSISTANT PROFESSOR OF SURGERY \\ D. BOYD BIGELOW \\ M.D. Colorado \\ ASSISTANT IN MEDICINE AND AMERICAN THORACIC SOCIETY-NATIONAL \\ TUBERCULOSIS ASSOCIATION FELLOW IN PULMONARY DISEASE \\ Thomas L. Petty \\ M.D. Colorado \\ ASSISTANT PROFESSOR OF MEDICINE \\ BERNARD E. LEVINE \\ M.D. Michigan \\ MEERICAN THORACIC SOCIETY-NATIONAL TUBERCULOSIS ASSOCIATION \\ FELLOW IN PULMONARY DISEASE* \\ From the Departments of Surgery and Medicine, \\ University of Colorado Medical Center, Denver, Colorado, U.S.A.
}

Figura 1. Portada del artículo original "Acute Respiratory Distress Syndrome in Adults", publicado en Lancet el 12 de agosto de 1967.

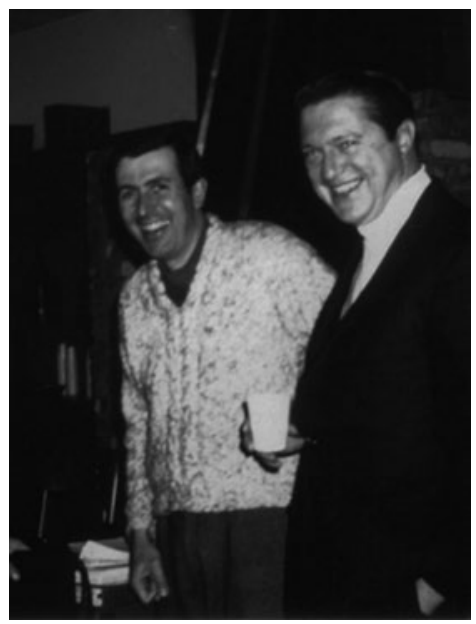

Figura 2. Los pioneros en la descripción y definición del síndrome de insuficiencia respiratoria aguda, los doctores Thomas L. Petti (saco negro) y David G. Ashbaugh (suéter blanco).

elevada incidencia, costos de atención y secuelas. Su patrón epidemiológico es cambiante y variable, dependiendo de la región que se evalúe, los recursos disponibles para su diagnóstico y tratamiento y el ámbito en el que se presenta, extrahospitalario o intrahospitalario. Lo que es una realidad es la complejidad de su comportamiento epidemiológico, evaluado en el estudio LUNG-SAFE, estudio multinacional que aportó datos fundamentales para entender mejor al SIRA y su comportamiento por regiones. La incidencia global es tan variable como de 3 a 80 entre 100000 habitantes, con una mortalidad que va de 15 
a $66 \%$. Estas cifras tan discordantes dependen de la región e institución evaluadas. ${ }^{2,3}$

\section{Definición}

EI SIRA es un tipo de insuficiencia respiratoria aguda secundaria a inflamación que resulta en incremento de la permeabilidad endotelial y lesión epitelial que condiciona paso de líquido al intersticio y a los sacos, incremento del cortocircuito intrapulmonar e hipoxemia refractaria a la oxigenoterapia convencional. Para entenderlo se tiene que partir de una definición basada en constructos, resultado de un complejo sustrato de interacciones genéticas, moleculares y celulares, que condicionan un buen número de manifestaciones que pueden evaluarse clínicamente y con estudios de laboratorio e imagen. La definición ha variado desde su descripción en 1967, se han hecho diversas modificaciones en las cuales han trabajado varios grupos hasta llegar a la Definición de Berlín, vigente en la actualidad. En este sentido, pueden destacarse varios momentos en la evolución de los criterios definitorios:

a) 1967: Ashbaugh et al. destacaron disnea, taquipnea, cianosis resistente a oxigenoterapia, disminución de la distensibilidad pulmonar, infiltrados alveolares difusos en la radiografía de tórax, atelectasias, congestión vascular, hemorragia, edema pulmonar y membranas hialinas.

b) 1988: Murray et al. ${ }^{4}$ establecieron una escala que incluye oxigenación, niveles de PPFE, distensibilidad del sistema respiratorio y extensión de los infiltrados pulmonares, evaluados por cuadrantes en la radiografía de tórax.

c) 1994: Bernard et al., ${ }^{5}$ a partir de la Conferencia de Consenso Americana-Europea, establecieron una definición basada en tres criterios que incluían la radiografía de tórax, el índice de oxigenación basado en la relación $\mathrm{PaO}_{2} / \mathrm{FiO}_{2}$ y la exclusión de insuficiencia cardiaca como causante de la congestión y edema pulmonar, de la que se derivaron los siguientes criterios:

- Aparición aguda de infiltrados pulmonares en la radiografía de tórax

- Presión en cuña de la arteria pulmonar < 19 mm Hg o ausencia de hipertensión de la aurícula izquierda

- Relación $\mathrm{PaO}_{2} / \mathrm{FiO}_{2}<300 \mathrm{~mm} \mathrm{Hg}$, con criterio de SIRA con una relación $\mathrm{PaO}_{2} / \mathrm{FiO}_{2}<200 \mathrm{~mm} \mathrm{Hg}$

d) Definición de Berlín. ${ }^{6} \mathrm{~A}$ partir de su publicación en 2012 es la utilizada para definir clínicamente la enfermedad. En relación con las definiciones previas elimina el término de lesión pulmonar aguda y el criterio de la presión capilar pulmonar, además de agregar los ajustes de ventilación a una PPFE $>5$. De esta definición fueron eliminados conceptos considerados clásicos en el SIRA, como la extensión de los infiltrados, la distensibilidad del sistema respiratorio, la PPFE $>10 \mathrm{~cm} \mathrm{H}_{2} \mathrm{O}$ y el volumen corregido espirado por minuto $(>10 \mathrm{~L} / \mathrm{m})$. A pesar de ser una definición más coherente, basada en una muestra grande y en evidencia científica, seguramente será mejorada en el futuro (Tabla 1).

Los autores hacen énfasis en que se debe descartar edema pulmonar cardiogénico y otras causas de insuficiencia respiratoria e infiltrados pulmonares y cumplir con los siguientes criterios:

- Inicio agudo en la primera semana de presentarse el disparador.

- Infiltrados pulmonares bilaterales en la radiografía de tórax o en la tomografía axial computarizada, no explicados por derrame pleural, atelectasias o nódulos pulmonares.

- Infiltrados no explicados por insuficiencia cardiaca o sobrecarga hídrica. Se puede requerir ecocardiograma para evaluar la función cardiaca.

- Deterioro de la oxigenación, definido por la relación $\mathrm{PaO}_{2} / \mathrm{FiO}_{2}$ (relación entre la presión arterial de oxígeno y la fracción inspirada de oxígeno) o por la relación $\mathrm{SaO}_{2} / \mathrm{FiO}_{2}$ (relación entre la pulsioximetría periférica y la fracción inspirada de oxígeno).

- El grado de hipoxemia define la gravedad y mortalidad asociada.

\section{Avances y contribuciones}

En 50 años se han logrado avances significativos en el conocimiento del SIRA relacionados con su biología molecular, fisiopatología, técnicas de imagen para su evaluación, escenarios clínicos, patrones de evolución, comportamiento hemodinámico, comunicación interorgánica, desenlaces, tratamiento farmacológico y ventilatorio.

En la descripción inicial del síndrome, los autores señalaron como características de la enfermedad la presencia de congestión pulmonar, atelectasias y daño alveolar difuso acompañado de edema y hemorragia alveolares. A partir de esta descripción inicial y con base en modelos animales y clínicos, se han comprendido las interacciones desencadenadas a partir de un disparador para activar una intrincada red 
Tabla 1. Definición de Berlín

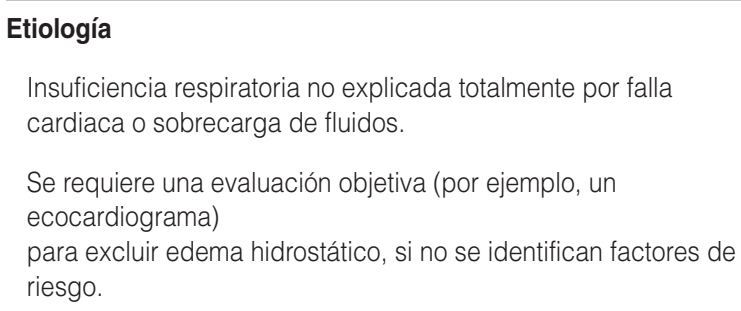

Insuficiencia respiratoria no explicada totalmente por falla cardiaca o sobrecarga de fluidos.

Se requiere una evaluación objetiva (por ejemplo, un ecocardiograma)

para excluir edema hidrostático, si no se identifican factores de riesgo.

\section{Tiempo de aparición}

En la semana de la identificación del disparador clínico o síntomas respiratorios nuevos que empeoran.

\section{Radiografía de tórax o tomografía axial computarizada}

Infiltrados bilaterales, no explicados totalmente por derrames, colapso lobar o pulmonar o nódulos.

\section{Alteración de la oxigenación}

Si la altitud es $>1000 \mathrm{~m}$, corregir $\mathrm{PaO}_{2} / \mathrm{FiO}_{2} \times(\mathrm{PB}$ barométrica/760) -SIRA leve: $200<\mathrm{PaO}_{2} / \mathrm{FiO}_{2} \leq 300$ con PPFE o PPCA $\geq 5 \mathrm{~cm} \mathrm{H} \mathrm{H}_{2} \mathrm{O}$ -SIRA moderado: $100<\mathrm{PaO}_{2} / \mathrm{FiO}_{2} \leq 200 \mathrm{con} \mathrm{PPFE} \geq 5 \mathrm{~cm} \mathrm{H}_{2} \mathrm{O}$ -SIRA grave: $\mathrm{PaO}_{2} / \mathrm{FiO}_{2} \leq 100$ con PPFE $\geq 5 \mathrm{~cm} \mathrm{H}_{2} \mathrm{O}$

$\mathrm{PaO}_{2}$, presión arterial de oxígeno; $\mathrm{FiO}_{2}$, fracción inspirada de oxígeno; PPFE, presión positiva al final de la espiración; PPCA, presión positiva continua en la vía aérea; SIRA, síndrome de insuficiencia respiratoria aguda.

de señalización molecular y celular que monta una intensa respuesta inflamatoria pulmonar y sistémica, base de la lesión alveolar a nivel endotelial, epitelial y del surfactante, sustrato fundamental del SIRA que correlaciona estrechamente con el contenido de agua intersticial y alveolar, el comportamiento clínico y mecánico, las diferentes fases evolutivas y los biomarcadores utilizados para el diagnóstico y seguimiento. La hipoxemia profunda y resistente a incremento en la fracción inspirada de oxígeno secundaria al colapso alveolar e incremento del cortocircuito intrapulmonar (Qs/Qt) es fundamental en la evolución clínica y fisiopatológica. ${ }^{7,8}$

Uno de los grandes avances en el conocimiento del SIRA es el concepto de "pulmón de bebe" (baby lung) desarrollado por el grupo de Gattinoni a partir de estudios de tomografía axial computarizada torácica y mecánica pulmonar. Representa el porcentaje de pulmón funcional y anatómicamente sano en el contexto anatómico y funcional del paciente con SIRA. Depende de la gravedad de la lesión y determina la distensibilidad del sistema respiratorio, la eliminación de $\mathrm{CO}_{2}$ y la oxigenación. Tiene un comportamiento diferente a la fracción del pulmón enfermo, pero tiene el riesgo de inflamarse y disfuncionar, en especial si hay sobrecarga de volumen, si se elige una inadecuada estrategia ventilatoria o si no se controla el disparador. Es importante mencionar que el SIRA no es una lesión homogénea sino heterogénea y de predominio basal, delimitándose tres áreas segmentarias alveolares, condensación, colapso y apertura. El concepto "pulmón de bebe" determinó el conocimiento y desarrollo de las estrategias fundamentales de manejo, entre las que destacan la ventilación con volúmenes corrientes bajos, la protección pulmonar, apertura alveolar y la posición en decúbito prono, entre otras. ${ }^{9,10-12}$

\section{Ventilación mecánica}

Hasta el momento ningún medicamento ha sido efectivo para prevenir o tratar el SIRA, si bien la ventilación mecánica se ha constituido en la piedra angular del tratamiento. Con base en el conocimiento de su fisiopatología y biología molecular se han desarrollado diversos modos de ventilación conforme el comportamiento celular y mecánico pulmonar; en especial se ha avanzado en el concepto de la lesión, que puede derivar en una inadecuada elección del modo de ventilación y que está relacionada con una excesiva presión transpulmonar 0 a modificaciones significativas en la presión pleural, ya sea positiva o negativa, que impactan en condicionar mayor inflamación, daño alveolar e inestabilidad hemodinámica. El monitoreo de la ventilación y el conocimiento en los cambios que presenta la mecánica pulmonar y torácica fueron clave para el desarrollo de los diferentes modos de ventilación y, en especial, su implementación con base en un protocolo personalizado.13-16

Conforme a evidencia científica se ha establecido que una ventilación mecánica con volúmenes corrientes bajos ( 6 a $8 \mathrm{~mL} / \mathrm{Kg}$ ), el mantenimiento de una presión meseta $<30 \mathrm{~cm} \mathrm{H}_{2} \mathrm{O}$ (en especial en $28 \mathrm{~cm} \mathrm{H}_{2} \mathrm{O}$ ), una adecuada titulación de la PPFE y la disminución del flujo inspiratorio, a la par de una estrategia protectora de ventrículo derecho, es la mejor opción para la supervivencia, en especial porque reducen el riesgo de desarrollar lesión pulmonar inducida por ventilación (LPIV). Enfermos con formas leves de SIRA pueden responder a ventilación no invasiva con CPAP o a otros modos de ventilatorios, entre los que destacan la ventilación de dos niveles (bi-level), la ventilación proporcional asistida y flujos altos de oxígeno. En las formas graves que cursan con hipoxemia resistente al manejo ventilatorio convencional se puede implementar la ventilación oscilatoria de alta frecuencia o la oxigenación extracorpórea de membrana, junto con otras medidas como el decúbito prono. ${ }^{17-20}$

Actualmente se promueven nuevos conceptos relacionados con la ventilación mecánica, sus potenciales 


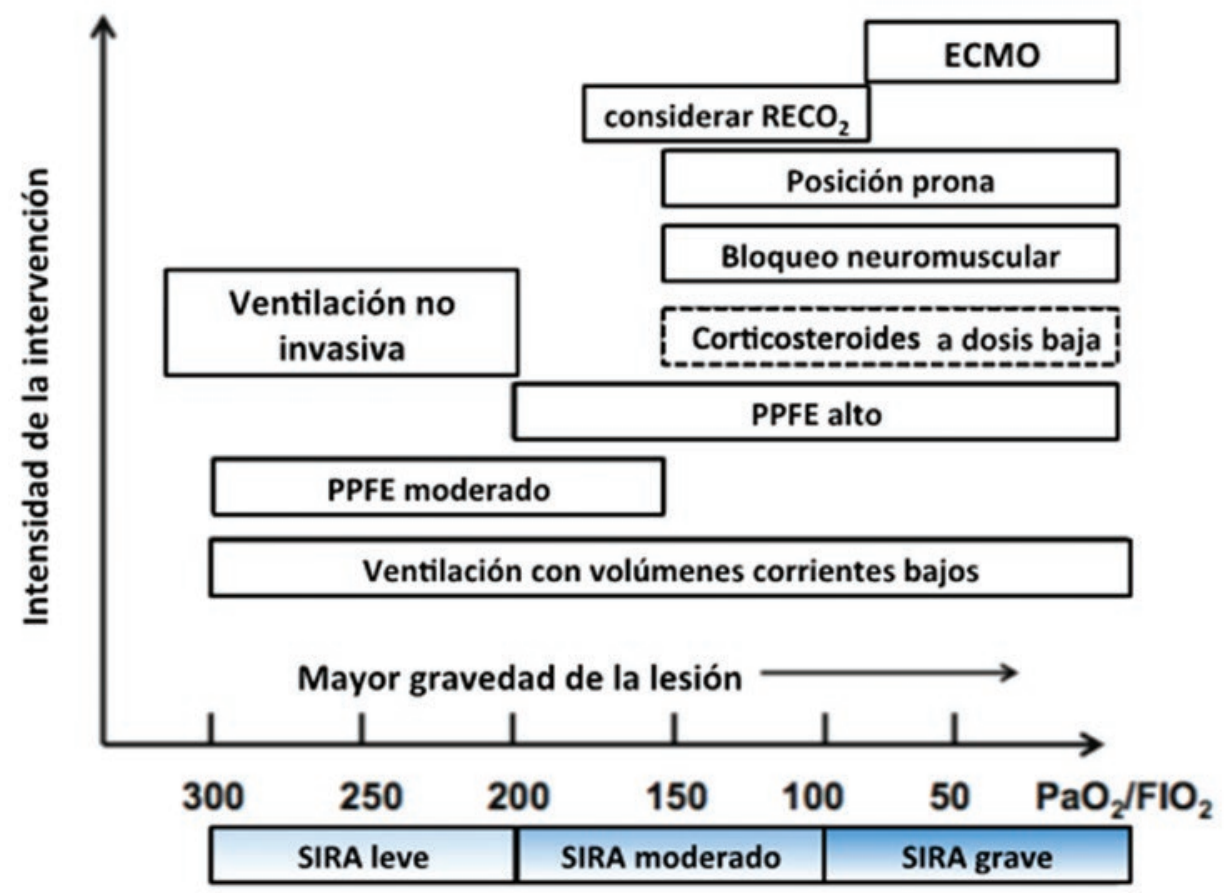

Figura 3. Estrategias de manejo con base en la gravedad del síndrome de insuficiencia respiratoria aguda. (SIRA). PPFE, presión positiva al final de la espiración; $\mathrm{RECO}_{2}$, removedor extracorpóreo de $\mathrm{CO}_{2}$; ECMO, oxigenación por membrana extracorpórea de oxígeno.

efectos deletéreos y pronóstico. Entre ellos destacan la presión de distensión y el poder mecánico, que establecen los límites de seguridad de la ventilación mecánica y ayudan a individualizar de forma dinámica la estrategia ventilatoria que asegure la menor lesión pulmonar y la mayor supervivencia (Figura 3). ${ }^{21,22}$

En conjunto con la ventilación mecánica se han implementado medidas adyuvantes para el manejo del SIRA como el uso de bloqueadores neuromusculares en las fases tempranas, que ha resultado en mejoría en la supervivencia, y de esteroides y prostaciclina en pacientes seleccionados. Es fundamental la combinación de una estrategia ventilatoria individualizada, manejo establecido con base en objetivos y el mantenimiento de un balance hídrico neutro o negativo conforme a una estrategia conservadora. Dado que la mayoría de los tratamientos farmacológicos han fracasado, se siguen intentado nuevas alternativas con potencial impacto en SIRA, entre ellas la aspirina y las células madre. ${ }^{23-29}$

En los últimos años se ha aprendido que el SIRA deja secuelas importantes en los pacientes que sobreviven, que limitan su calidad de vida y elevan los costos de atención; entre estas son dignas de mencionar la neuropatía, miopatía, sarcopenia, contracturas musculares, disfunción neurocognitiva y síndrome de estrés postraumático, que pueden persistir hasta cinco años después del diagnóstico a pesar del tratamiento y la rehabilitación. Por este motivo se están desarrollando estudios relacionados con diferentes escenarios en este sentido, procesos de manejo multidisciplinario y programas de información dirigidos a los pacientes y sus familiares. ${ }^{30,31}$ 


\title{
Tratamiento del síndrome de insuficiencia respiratoria aguda
}

\author{
Gilberto Felipe Vázquez-De Anda, Cynthia Ivonne Mejía-Pérez, María Guadalupe Delaye-Aguilar, \\ Ana Ivonne Pérez-Castañeda
}

\section{Introducción}

El 12 de octubre de 1967, Ashbaugh, Bigelow, Petty y Levine describieron en 12 pacientes adultos lo que en un principio se denominaría el síndrome de insuficiencia respiratoria progresiva del adulto (SIRPA). ${ }^{1}$ La descripción clásica del síndrome concatena signos de insuficiencia respiratoria bajo un solo proceso fisiopatológico similar al observado en neonatos que presentan el síndrome de "membranas hialinas", una variedad de insuficiencia respiratoria observada en casos de deficiencia de surfactante alveolar. Hace 50 años, la descripción de lo que hoy en día se denomina síndrome de insuficiencia respiratoria aguda (SIRA) reunió en una sola entidad aquellos eventos que habían sido descritos bajo múltiples denominaciones como "pulmón de choque", "pulmón de Na-gang", "pulmón húmedo", insuficiencia respiratoria por embolia grasa. Estas entidades se caracterizaban por estar asociadas con una acometida al tejido pulmonar de forma directa $o$ indirecta, el cual respondía con un proceso inflamatorio en el epitelio alveolar y al capilar pulmonar. ${ }^{1,32}$

El punto de impacto y base del proceso fisiopatológico del SIRA es el alveolo (unidad anatomo-funcional del pulmón) involucrando sus tres espacios: intraalveolar, intersticial y vascular (endotelial), cuya manifestación es la hipoxemia grave, infiltrados pulmonares y edema pulmonar de origen no cardiovascular. ${ }^{32}$ Este proceso dinámico altera las propiedades del alveolo en la interface aire-líquido, permitiendo el ingreso al espacio alveolar de líquidos y proteínas provenientes del espacio intersticial. Este edema rico en proteínas altera las propiedades del surfactante alveolar con la consecuente inactivación de este. El surfactante inactivado incrementa la tensión de superficie alveolar llevando al colapso alveolar, promoviendo el incremento de cortocircuitos y disminución de la oxigenación arterial. ${ }^{32}$

Aunado a lo anterior, el colapso arterial incrementa la fricción entre los alveolos adyacentes difundiendo el proceso inflamatorio, cuya evolución desencadena como producto final fibrosis que ocasiona pérdida de la función de los alveolos afectados. Entonces, a mayor número de alveolos involucrados, mayor el colapso alveolar y sus consecuencias: hipoxemia, baja distensibilidad pulmonar, incremento en los cortocircuitos intrapulmonares, capacidad funcional disminuida, atelectasias y edema pulmonar no cardiogénico.

En el transcurso de 50 años, el tratamiento del SIRA se ha enfocado en atender cada una de las fases del proceso fisiopatológico. En su descripción original, Ashbaugh et al. apuntaron que la ventilación mecánica es la medida terapéutica más importante para contrarrestar los efectos del colapso alveolar mediante la ventilación mecánica a presión positiva para reclutar los alveolos colapsados y el uso de la presión positiva al final de la espiración (PEEP), para evitar el colapso de las unidades alveolares funcionales. ${ }^{1}$ Actualmente se sabe que la ventilación por sí misma puede condicionar daño al pulmón, incluso mayor al producido por la causa primaria, por eso deben utilizarse medidas encaminadas a proporcionar una ventilación protectora. ${ }^{33}$ Además, entre otras estrategias terapéuticas se encuentra disminuir o limitar el proceso inflamatorio y mantener el espacio alveolar libre de líquido., ${ }^{1,32-34}$

\section{Medidas para contrarrestar el proceso inflamatorio}

El daño pulmonar puede ser inducido de manera directa (por ejemplo, neumonía) o indirecta (por ejemplo, sepsis abdominal), lo que desencadena un proceso inflamatorio con las consecuencias descritas. Por ello, en primer término, se debe tratar la causa que originó al daño pulmonar. El inicio temprano de antibióticos en los casos de sepsis grave, drenado de abscesos o colecciones en cavidades, estabilidad hemodinámica, atención de fracturas en los casos de trauma grave son ejemplo de las medias primarias en el SIRA. ${ }^{35}$ El uso de altos volúmenes pulmonares al final de la inspiración, combinado con altas presiones en la vía aérea y bajos niveles de PEEP favorecen la sobredistensión alveolar (con daño subsecuente a la interface aire-líquido) y el fenómeno de apertura-colapso (que produce lesión directa de las paredes alveolares por cizallamiento). Debido a lo anterior, es 
imperioso promover una ventilación mecánica protectora desde el inicio mediante bajas presiones de meseta $\left(<30 \mathrm{~cm} \mathrm{H} \mathrm{H}_{2} \mathrm{O}\right.$ ), favorecer un volumen corriente bajo de $6 \mathrm{~mL} / \mathrm{kg}$ de peso corporal (calculado $50 \pm 0.91$ [estatura en centímetros - 152.4] para hombres y $45.5 \pm 0.91$ [estatura en centímetros - 152.4] para mujeres), con un ciclado a bajas presiones diferenciales (menor a $16 \mathrm{~cm} \mathrm{H}_{2} \mathrm{O}$ ) y niveles apropiados de PEEP (8 a $10 \mathrm{~cm} \mathrm{H}_{2}$ O). . $^{33,34,36}$

El uso de esteroides para controlar el proceso inflamatorio pulmonar y los cambios secundarios al proceso de fibrosis han sido documentados desde la descripción original del SIRA, sin embargo, hasta la fecha su uso es controvertido y los expertos consideran que los esteroides deben emplearse solo en casos especiales. ${ }^{33,37}$

Se ha descrito que, entre otros factores, la hipoxemia grave resistente a tratamiento con oxígeno suplementario observada en el SIRA se relaciona con la vasoconstricción por hipoxia, con la hipertensión pulmonar y las alteraciones en la relación ventilación-perfusión. Por lo anterior, se pensó que las propiedades del óxido nítrico como agente vasodilatador y antiinflamatorio podría ser utilizado como medida terapéutica en el SIRA. Sin embargo, los resultados de estudios clínicos y metaanálisis recientes no mostraron efecto en la disminución de la mortalidad en los diferentes niveles de hipoxemia a pesar de mejorar la oxigenación de 7 a $16 \%$. Tampoco ha demostrado que disminuya el costo por hospitalización o la estancia en la unidad de cuidados intensivos. Por lo anterior, debe ser utilizado de manera racional y en casos específicos. . $^{33,34,38}$

\section{Medidas para disminuir el edema agudo pulmonar de origen no cardiovascular}

La descripción de edema agudo pulmonar de origen no cardiovascular fue uno de los principales hallazgos descritos por Ashbaugh et al. ${ }^{1}$ Los autores describieron los infiltrados bilaterales en la radiografía de tórax. El origen del edema pulmonar se debe a incremento en la permeabilidad vascular secundaria al proceso inflamatorio endotelial, a disminución del desplazamiento de líquido por el intersticio alveolar y al fenómeno de apertura y colapso, que promueven un efecto de succión durante el colapso alveolar que favorece el desplazamiento de líquido rico en proteínas proveniente del capilar pulmonar e intersticio hacia la luz alveolar. ${ }^{32}$
El uso de albúmina y balances neutros o negativos han sido promovidos por más de 50 años. Sakr et al. ${ }^{39}$ describieron la asociación entre el balance de líquidos y mortalidad en pacientes con lesión pulmonar aguda-insuficiencia respiratoria aguda. En su estudio encontraron que los pacientes con insuficiencia respiratoria aguda se asociaron con balance de líquidos a las 96 horas más positivo que los pacientes sin lesión pulmonar aguda. Además, se estableció la asociación entre el balance positivo de líquidos y mayor mortalidad. ${ }^{39}$ Martin et al. ${ }^{40}$ trataron a pacientes con lesión pulmonar aguda (LPA)/SIRA con albumina y furosemida comparando este tratamiento con el manejo convencional. Los autores documentaron que al tercer día de tratamiento los pacientes que recibieron albumina y furosemida tuvieron mayor $\mathrm{PaO}_{2} / \mathrm{FiO}_{2}$, mayor concentración sérica de proteínas totales y balances negativos.

El Grupo Colaborativo para el Tratamiento de la LPA/SIRA (ARDS Network), con la participación de múltiples hospitales en Estados Unidos, comparó a un grupo con tratamiento liberal de líquidos y diuréticos con un grupo cuyo manejo fue conservador manteniendo un balance hídrico neutro o negativo. Los resultados mostraron que el grupo conservador recibió mayores dosis de furosemida y obtuvo balances más negativos del primer al séptimo día en comparación con el grupo liberal. Además, se observó en el grupo conservador menos días en ventilación mecánica, menos días en terapia intensiva, menos días de estancia hospitalaria y menos días con disfunción orgánica. Sin embargo, no se observaron diferencias en la mortalidad. ${ }^{41}$

\section{Medidas encaminadas a contrarrestar el colapso alveolar}

La ventilación mecánica ha sido la medida más efectiva para contrarrestar los efectos del colapso alveolar. Sin embargo, se ha demostrado que por sí misma puede producir daño alveolar cuando no se usan medidas protectoras que prevengan o limiten el daño pulmonar inducido por la ventilación mecánica. La ventilación protectora se caracteriza por proporcionar una ventilación con altos volúmenes pulmonares, un ciclado con volumen corriente bajo, bajas presiones inspiratorias, bajas presiones diferenciales durante el ciclado y adecuados niveles de PEEP (Figura 4). Brochard et al. ${ }^{42}$ realizaron un estudio clínico comparativo a dos diferentes dosis de volumen corriente: un grupo recibió ventilación protectora con un volumen corriente bajo y 


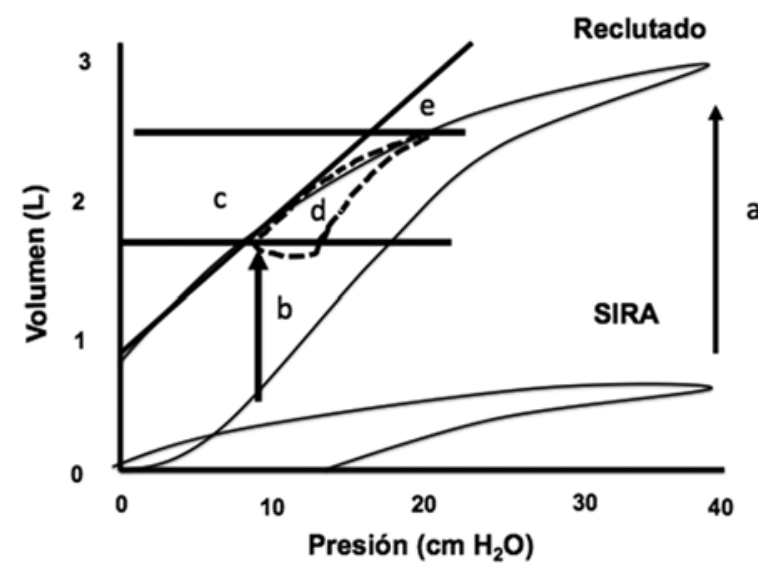

Figura 4. Curva de presión-volumen en la que se muestra el concepto de ventilación protectora durante la ventilación mecánica. a) Maniobra de reclutamiento. b) Alto volumen pulmonar al final de la espiración. c) NIVEL de PEEP para evitar colapso alveolar al final de la espiración y pérdida de volumen pulmonar. d) Ciclado con volumen corriente bajo a bajas presiones diferenciales. e) Bajas presiones meseta para evitar sobredistensión alveolar al final de la inspiración (para más detalles ver texto).

otro recibió un volumen corriente alto, ambos grupos con presiones inspiratorias menores a $30 \mathrm{~cm} \mathrm{H}_{2} \mathrm{O}$. Los resultados mostraron que no hubo diferencias en la mortalidad entre los grupos tratados.

En el mismo periodo, Stewart et al. ${ }^{43}$ realizaron un estudio cuyo objetivo fue determinar el beneficio del uso de volúmenes corrientes de $7 \mathrm{~mL} / \mathrm{kg}$ versus $10 \mathrm{~mL} / \mathrm{kg}$ durante la ventilación mecánica. Los resultados no mostraron diferencias en la mortalidad en ambos grupos. Al igual que el estudio anterior se observó que ambos grupos mantuvieron presiones máximas menores a $30 \mathrm{~cm} \mathrm{H}_{2} \mathrm{O}$. Brower et al. ${ }^{44}$ realizaron un estudio comparativo en el que administraron dos niveles volúmenes corrientes. Los resultados no mostraron diferencias en la mortalidad. Al igual que los estudios anteriores, las presiones meseta se mantuvieron por debajo de los $30 \mathrm{~cm} \mathrm{H}_{2} \mathrm{O}$. Amato et al. ${ }^{45}$ realizaron un estudio comparativo de pacientes con insuficiencia respiratoria: los expusieron a volúmenes corrientes bajos, presiones meseta bajas y adecuados niveles de PEEP posterior a una maniobra de reclutamiento. En este estudio se determinó una diferencia significativa en la mortalidad, donde el grupo que recibió ventilación protectora tuvo mejor supervivencia. El ARDS Network ${ }^{46}$ realizó un estudio clínico multicéntrico en el que se compararon dos modos de ventilación. Un grupo recibió ventilación protectora versus ventilación convencional, con un volumen corriente alto. En este estudio se documentó que una ventilación con $12 \mathrm{~mL} / \mathrm{kg}$ de volumen corriente está asociado con mayor mortalidad comparado con los pacientes con ventilación protectora a quienes se les proporcionó un volumen corriente de $6 \mathrm{~mL} / \mathrm{kg}$. Además, se observó que los pacientes con ventilación protectora mantuvieron presiones máximas menores a $30 \mathrm{~cm} \mathrm{H} \mathrm{H}_{2}$; los volúmenes corrientes $\geq 12 \mathrm{~mL} / \mathrm{kg}$ se asociaron con mayor mortalidad (Tabla 2). En un metaanálisis realizado por Burns et al. ${ }^{47}$ se determinaron un factor de protección a favor de las medidas protectoras de ventilación mecánica caracterizadas por bajos volúmenes corrientes y bajas presiones máximas.

En 1992, Lachman propuso la protección pulmonar mediante la reapertura de unidades alveolares colapsadas a través de maniobras de reclutamiento pulmonar. En su editorial "Abrir el pulmón y mantenerlo abierto"48 recomienda re-aerear activamente las unidades alveolares y mantenerlas abiertas con suficientes niveles de PEEP, evitando el colapso alveolar al final de la espiración, así como promover un ciclado ventilatorio con bajas presiones diferenciales y promover la protección pulmonar a través de la preservación del surfactante pulmonar. El objetivo del tratamiento y la prevención de la insuficiencia respiratoria aguda se basa en tres aspectos: la reapertura de unidades alveolares colapsadas, preservar el componente activo de surfactante en las unidades alveolares que se mantienen funcionales y prevenir el colapso alveolar al final de la espiración.

Meade et al. ${ }^{49}$ realizaron un estudio multicéntrico; observaron el beneficio de una ventilación mecánica protectora después de una maniobra de reclutamiento alveolar. Los resultados mostraron que los pacientes con apertura pulmonar tuvieron menor hipoxemia resistente, menor mortalidad por hipoxemia y menor uso de terapias de rescate. Sin embargo, no se observaron diferencias significativas en la mortalidad. Recientemente, Amato ${ }^{50}$ mostró en un estudio comparativo de pacientes con cirugía cardiovascular que quienes recibían una maniobra de reclutamiento completa o agresiva tienen menor mortalidad, complicaciones posoperatorias y menor hipoxemia, que los pacientes que reciben una dosis menos agresiva de reclutamiento pulmonar.

El colapso alveolar es una consecuencia del proceso inflamatorio que promueve la permeabilidad alveolar con infiltrado a la luz alveolar de líquido rico en proteínas, inactivando el surfactante pulmonar y promoviendo cambios en la tensión de la superficie alveolar que finalmente llega al colapso. Adicional a las alteraciones del sistema de surfactante se encuentra 
Tabla 2. Estudios clínicos en los que se comparó el beneficio de medidas de protección pulmonar versus terapia convencional

\begin{tabular}{|c|c|c|c|c|c|c|c|c|c|c|}
\hline \multirow[t]{2}{*}{ Estudio (referencia) } & \multicolumn{2}{|c|}{ No. pacientes } & \multicolumn{2}{|c|}{$\begin{array}{l}\text { Media del } \\
\text { Vt medido } \\
(\mathrm{mL} / \mathrm{kg})\end{array}$} & \multicolumn{2}{|c|}{$\begin{array}{c}\text { Media de la presión } \\
\text { meseta } \\
\left(\mathrm{cm} \mathrm{H} \mathrm{H}_{2} \mathrm{O}\right)\end{array}$} & \multicolumn{2}{|c|}{$\begin{array}{l}\text { Media del PEEP } \\
\qquad\left(\mathrm{cm} \mathrm{H}_{2} \mathrm{O}\right)\end{array}$} & \multicolumn{2}{|c|}{$\begin{array}{l}\text { Mortalidad } \\
\quad(\%)\end{array}$} \\
\hline & Exp. & Control & Exp. & Control & Exp. & Control & Exp. & Control & Exp. & Control \\
\hline Brochard ${ }^{42}$ & 58 & 58 & 7.1 & 10.3 & 25.7 & 31.7 & 9.6 & 8.5 & 46.6 & 37.9 \\
\hline Stewart ${ }^{43}$ & 60 & 60 & 7.2 & 10.8 & 22.3 & 26.8 & 8.6 & 7.2 & 50 & 47 \\
\hline Brower ${ }^{44}$ & 26 & 26 & 7.3 & 10.2 & 24.9 & 30.6 & NA & NA & 50 & 46 \\
\hline Amato 45 & 29 & 24 & 6.1 & 11.6 & 23.7 & 37.8 & 13.2 & 9.3 & 38 & 71 \\
\hline ARDS Network ${ }^{46}$ & 432 & 429 & 6.2 & 11.8 & 25 & 36 & 8.1 & 9.1 & 31 & 39.8 \\
\hline
\end{tabular}

Vt, volumen corriente; Exp., grupo experimental; Control, grupo control.

la presión sobreimpuesta que promueve el colapso principalmente en las zonas posteriores. El resultado del colapso de unidades alveolares es la pérdida de volumen intraalveolar: a medida que se agregan unidades alveolares colapsadas disminuye la capacidad funcional residual, promoviendo zonas atelectasicas y la aparición de cortocircuitos intrapulmonares de derecha a izquierda con la consecuente hipoxemia. En la descripción original del síndrome, ${ }^{1}$ los autores recomiendan el uso de PEEP para mantener las unidades alveolares abiertas y evitar el colapso de estas.

A pesar del efecto fisiológico del PEEP, estudios clínicos controlados no han demostrado un efecto de protección y disminución de la mortalidad. En 1998, Amato et al., ${ }^{45}$ en su estudio demostraron que altos niveles de PEEP asociados con bajos volúmenes corrientes posteriores a una maniobra de reclutamiento y bajas presiones diferenciales están asociados con mayor oxigenación y menor mortalidad. El ARDS Network realizó un estudio comparativo entre dos grupos con diferentes niveles de PEEP en donde no se observaron diferencias significativas entre los grupos en relación con la mortalidad. ${ }^{51}$ En 2008, Mercat et al. ${ }^{52}$ realizaron un estudio multicéntrico en el que compararon dos grupos con insuficiencia respiratoria aguda: los pacientes que recibieron medidas de reclutamiento pulmonar requirieron menor cantidad de volumen intravascular, menor número de maniobras de rescate (posición prona, óxido nítrico, entre otras); los pacientes que recibieron niveles altos de PEEP tuvieron menos días en ventilación mecánica y menos días de disfunción orgánica.

Briel et al. ${ }^{53}$ realizaron un metaanálisis respecto al uso de niveles de PEEP comparando grupos que recibieron PEEP alto versus PEEP bajo con un volumen corriente de $6 \mathrm{~mL} / \mathrm{kg}$. Los autores reportaron que los pacientes con PEEP alto presentaron presiones meseta mayores que los pacientes con PEEP bajo, menores niveles de $\mathrm{FiO}_{2}$ y mejor oxigenación. Los autores documentaron mayor supervivencia al tiempo de hospitalización, 60 días, en los pacientes con insuficiencia respiratoria aguda que recibieron aleatoriamente altos niveles de PEEP. Además, documentaron que los pacientes con insuficiencia respiratoria al día 28 tuvieron más días sin apoyo ventilatorio que los pacientes con niveles bajos de PEEP.

Amato et al. ${ }^{21}$ realizaron un estudio para determinar el efecto de la presión diferencial en pacientes con insuficiencia respiratoria aguda. El estudio incluyó los resultados de pacientes que participaron en los estudios de insuficiencia respiratoria. Los autores establecieron que la mortalidad hospitalaria se incrementa cuando los pacientes presentan un nivel de PEEP constante con incrementos en la presión diferencial, caracterizados por altos niveles de presión meseta; en los pacientes con niveles constantes de presión diferencial e incrementos en el nivel de PEEP no se observó asociación con mortalidad. Finalmente, se observó disminución en la mortalidad en los pacientes en quienes el PEEP se incrementó y la presión diferencial se redujo. Se estableció que por cada $7 \mathrm{~cm}$ $\mathrm{H}_{2} \mathrm{O}$ por arriba de $15 \mathrm{~cm} \mathrm{H}_{2} \mathrm{O}$ de presión diferencial, se observó incremento en el riesgo de muerte.

Recientemente, Guérin et al. ${ }^{54}$ realizaron un estudio post hoc de pacientes con insuficiencia respiratoria incluidos en dos estudios aleatorizados. Los autores reportaron asociación significativa entre el incremento de la presión diferencial y la mortalidad al día uno.

La ventilación de alta frecuencia oscilatoria (VAFO) es un modo ventilatorio que por sus características de desempeño (muy bajos volúmenes corrientes, frecuencia respiratoria cercanas a 600 por minuto, altas presiones en la vía aérea y bajas presiones diferenciales) se adapta al concepto de ventilación protectora. 
Recientemente se reportaron dos estudios clínicos aleatorizados ${ }^{5,56}$ en los que se comparó ventilación convencional contra VAFO. En el estudio multicéntrico OSCILLATE, Ferguson et al. mostraron que los pacientes que recibieron VAFO tuvieron mayor mortalidad que quienes recibieron ventilación convencional protectora. En el segundo estudio multicéntrico OSCAR, Young et al. ${ }^{56}$ demostraron que los pacientes que recibieron VAFO no presentaron diferencia en mortalidad comparados con los pacientes tratados con ventilación convencional protectora. Recientemente, un consenso de expertos no recomienda el uso de la VAFO como medida terapéutica inicial para el manejo de la insuficiencia respiratoria aguda.

Guérin et al. ${ }^{18}$ realizaron un estudio clínico para determinar el beneficio del uso de la posición prona en pacientes con insuficiencia respiratoria aguda. El estudio demostró que los pacientes con hipoxemia grave que son colocados en posición prona por más de 12 horas tienen mejor supervivencia que los pacientes en posición supina. La mortalidad reportada es la menor registrada en estudios clínicos.

\section{Medidas de rescate}

En el transcurso de 50 años, la mortalidad por SIRA continúa siendo alta. Los reportes en la literatura varían entre 40 y $90 \%$ dependiendo del país, hospital y periodo en el que se detalla la mortalidad. ${ }^{33} \mathrm{La}$ hipoxemia y la disfunción orgánica múltiple son las principales causas de deceso, por eso se deben instalar medidas terapéuticas de "rescate" tan pronto como las medidas convencionales de ventilación protectora han fallado para restablecer el intercambio de gases y limitar el daño multiorgánico. El objetivo de estas medidas es evitar la muerte por hipoxemia y la progresión a disfunción orgánica múltiple.

La VAFO continúa vigente como una medida de rescate cuando la ventilación convencional ha fallado en mantener un adecuado intercambio de gases con parámetros de protección pulmonar.

Mehta et al. ${ }^{57}$ demostraron en un estudio comparativo que los pacientes que reciben VAFO como medida de rescate tienen mejor supervivencia que quienes continuaron con ventilación convencional. Si bien la VAFO no se recomienda como una medida terapéutica de inicio para el tratamiento del SIRA, debe establecerse cuando la ventilación convencional ha fallado. El desempeño del ventilador de alta frecuencia oscilatoria permite proporcionar una ventilación con bajos volúmenes corrientes a altas presiones en la vía aérea y bajas presiones diferenciales, ideales para un pulmón con baja distensibilidad pulmonar. La VAFO es una medida terapéutica temporal, por lo que una vez que se ha logrado estabilizar la función pulmonar a menor presión media de la vía aérea se debe iniciar la transición a ventilación convencional.

La sustitución de la función pulmonar con el intercambio de gases con membrana extracorpórea (ECMO, extracorporeal membrane oxigenation) es una medida avanzada de rescate para evitar la muerte por hipoxemia. El desarrollo y simplificación de las bombas extracorpóreas para mantener una adecuada perfusión, así como el desarrollo de mejores y más simplificadas membranas de intercambio gaseoso y el acceso veno-venoso con catéteres de alta seguridad han permitido mejorar el uso más eficiente, práctico y seguro de estos dispositivos. ${ }^{58}$ Los nuevos dispositivos para proporcionar ECMO han tenido mejores resultados que las experiencias con los dispositivos de primera generación. La pandemia de influenza AH1N1 dio la oportunidad de utilizar estos dispositivos como una medida avanzada de rescate de la función pulmonar en pacientes con neumonía grave. ${ }^{58}$ Actualmente existe un número limitado de estudios clínicos que permitan evaluar la eficacia y seguridad de esta medida terapéutica. Peek et al. ${ }^{59}$ realizaron un estudio clínico comparando el uso de ECMO contra terapia convencional en pacientes con SIRA grave. El estudio CESAR, que incluyó a 180 pacientes, no mostró diferencias en la mortalidad. El uso de ECMO debe ser iniciado cuando las demás medidas han fallado. Sin embargo, el inicio tardío de ECMO puede que no dé los resultados esperados.

Otros tipos de intercambio de gases por membrana extracorpórea están en investigación. Los intercambiadores veno-venoso de $\mathrm{CO}_{2}$ a flujo bajo permiten, en teoría, proporcionar medidas ultraprotectoras de ventilación mecánica al permitir la administración de volumen corriente a muy baja dosis (menor a $4 \mathrm{~mL}$ / $\mathrm{kg}$ ) y a muy bajas presiones máximas. El estudio clínico del uso de esta medida está en proceso..$^{60,61}$

\section{Conclusión}

A 50 años de la descripción del SIRA, la mortalidad por este síndrome continúa siendo alta. El tratamiento está enfocado en limitar y disminuir el proceso inflamatorio, el edema pulmonar y el daño inducido por la ventilación mecánica a través de medidas protectoras durante la ventilación mecánica. 


\title{
Fisiopatología del síndrome de insuficiencia respiratoria aguda
}

\author{
Jesús Carlos Briones-Garduño
}

La fisiopatología del síndrome de insuficiencia respiratoria aguda implica la ruptura de la barrera alveolocapilar, resultado de complejas interacciones entre células activadas, respuesta humoral y mediadores celulares. Después de la lesión inicial (endotoxemia, por ejemplo), se activan las células proinflamatorias con síntesis y secreción de mediadores inflamatorios, acumulación de polimorfonucleares, células fagocíticas que forman parte de la primera línea de defensa del organismo y normalmente se encuentran en la circulación sanguínea, pero cuando se presenta un proceso infeccioso-inflamatorio migran al sitio lesionado mediante quimiotaxis a través de la interleucina (IL-8). Mediante receptores específicos estas células reconocen microrganismos opsonizados y no opsonizados para facilitar su captura, causando la muerte de los microrganismos fagocitados por la acción de los radicales libres de oxígeno (anión superóxido, radicales hidroxilo, peróxido de hidrógeno, producidos por el complejo enzimático NADPH oxidasa) o del nitrógeno (óxido nítrico), así como otras moléculas como la mieloperoxidasa.

Cabe señalar que estas células liberan de manera progresiva sustancias preformadas contenidas en su gránulos (lipocalina, lisozima, LL-37 y otras como metaloproteinasas MP8, MMP9 y MMP25), que inducen la formación de NET (trampas extracelulares de neutrófilos) descritos en 2004 por Brinkman, los cuales funcionan como una barrera física para evitar diseminación y daño tisular, regulando diversas infecciones, así como limitando el proceso inflamatorio, el cual activa la cascada del complemento, provocando liberación de metabolitos del ácido araquidónico, citocinas (IL-1,IL6, IL-10, TNF $\alpha$, enzimas proteolíticas y radicales libres de oxígeno; simultáneamente, se magnifica la respuesta inflamatoria, con la activación de monocitos, macrófagos alveolares y plaquetas con incremento en su adhesividad y agregabilidad, provocando microtrombosis vascular e isquemia secundaria local y sistémica, lo que explica la falla orgánica múltiple que sucede en muchos casos (Figuras 5 y 6).62-64

Los pulmones dañados por este mecanismo requieren apoyo con ventilación mecánica, que puede contribuir con la lesión por cuatro procesos adicionales:
- Barotrauma (lesión por altas presiones o aire extra alveolar).

- Volutrauma (lesión por altas presiones transpulmonares y sobre distensión).

- Atelectrauma (colapso de alveolos inestables secundarios al cierre/apertura cíclicos con estiramiento de regiones sanas).

- Biotrauma (por alteración humoral).

Las fuerzas físicas del ventilador mecánico alteran la estructura y elasticidad pulmonar, ya que en pulmones lesionados el patrón de inflación es heterogéneo y la fuerza de distensión alveolar puede alcanzar hasta $140 \mathrm{~cm} \mathrm{H} \mathrm{H}_{2} \mathrm{O}$, aun con presiones transpulmonares de $30 \mathrm{~cm} \mathrm{H}_{2} \mathrm{O}$, provocando daño cíclico por fuerzas de cizallamiento resultantes del colapso y sobredistensión repetitivos que favorecen la translocación de mediadores, endotoxinas y bacterias desde el pulmón a la circulación sistémica. ${ }^{65}$

En la evolución clínica-patológica del SIRA se reconocen clásicamente tres fases:

- Fase exudativa: Hay ruptura de la membrana alveolo-capilar, con la consecuente acumulación de edema alveolar rico en proteínas y citocinas. La fase exudativa dura aproximadamente siete días y está clínicamente caracterizada por disnea, taquipnea e hipoxemia severa.

- Fase proliferativa: Si no existe recuperación, algunos pacientes desarrollarán progresivamente daño pulmonar y presentarán evidencia de inflamación pulmonar intersticial y fibrosis. Esta fase dura entre siete y 21 días.

- Fase fibrótica: Aunque la mayoría de los pacientes se recuperan entre tres y cuatro semanas después del daño inicial, algunos presentan fibrosis progresiva, por lo que requieren soporte ventilatorio prolongado, lo que los predispone a las complicaciones observadas en las unidades de cuidados intensivos. Diversos estudios apoyan la teoría de que esta fase final del SIRA se provoca por el uso de la ventilación mecánica con volumen o presión elevada.

La estructura alveolocapilar normal provee una gran superficie de intercambio gaseoso y una barrera estrecha entre el gas alveolar y la sangre de los capilares 


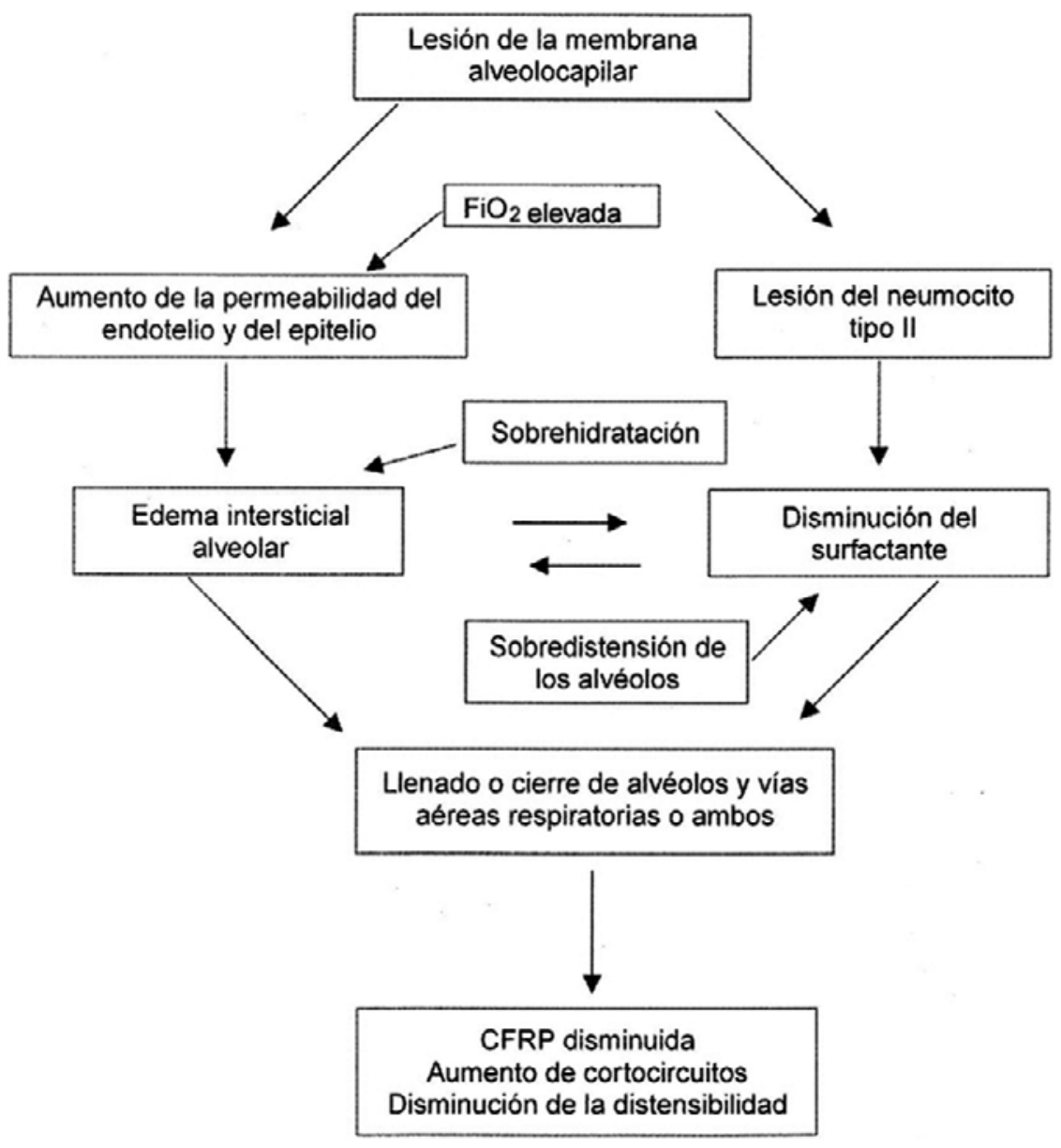

Figura 5. Fisiopatología del SIRA.

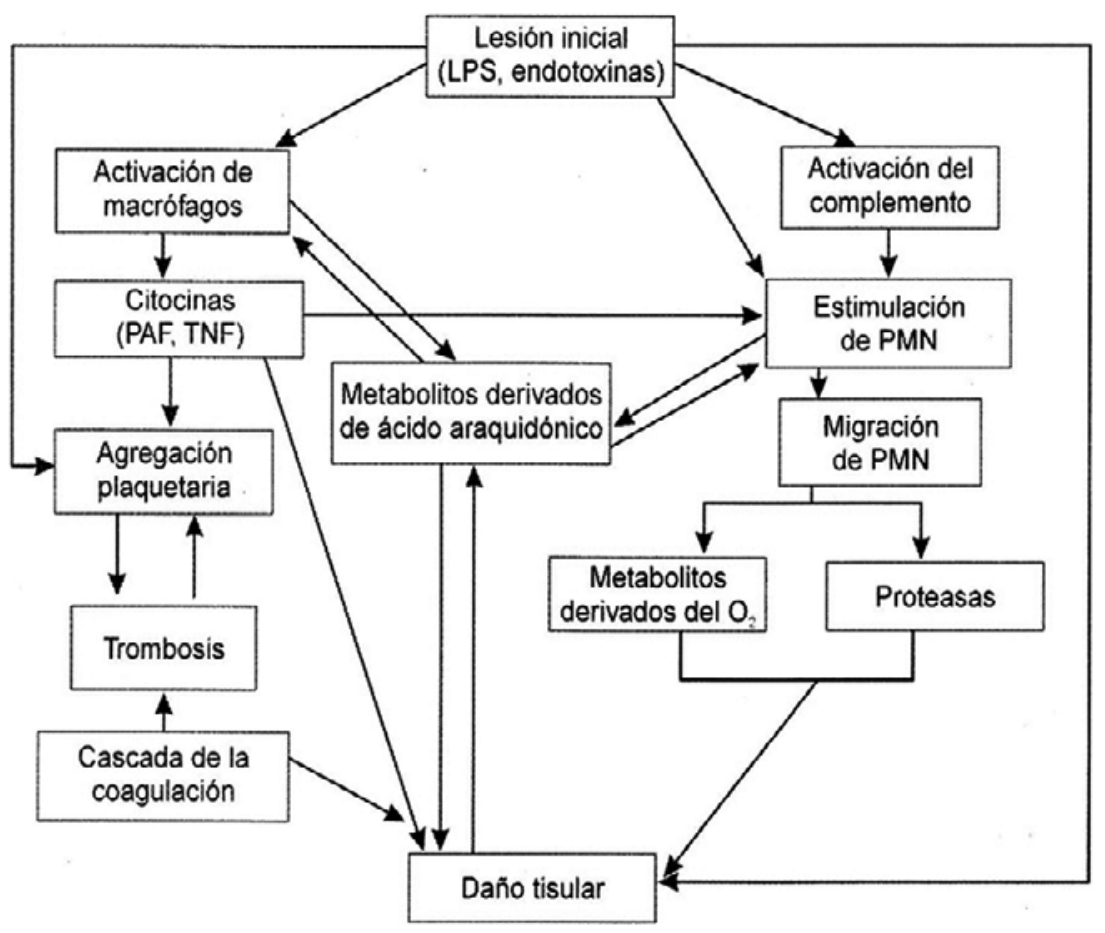

Figura 6. Mecanismo de daño tisular. 
pulmonares. El daño difuso de la región alveolar ocurre durante la fase aguda o exudativa de la lesión pulmonar del SIRA. Este daño involucra el tejido endotelial y el epitelial y altera la barrera pulmonar, inundando los espacios alveolares con líquido, lo que inactiva el surfactante, causando inflamación y produciendo alteraciones en el intercambio gaseoso. Estos eventos se reflejan en la presencia de infiltrados bilaterales, los cuales son indistinguibles por radiología convencional de los de origen cardiogénico.

Los hallazgos patológicos consisten en daño alveolar difuso, incluyendo lesión capilar. Los espacios alveolares tienen membranas hialinas y están llenos de edema rico en proteínas y células inflamatorias. El espacio intersticial, alveolos, pequeños vasos y capilares también contienen macrófagos, neutrófilos y eritrocitos. La fase aguda puede resolverse o progresar a fibrosis con hipoxemia persistente, aumento del espacio muerto, hipertensión pulmonar y pérdida de la distensibilidad. Las radiografías de tórax muestran nuevas opacidades lineales consistentes con la fibrosis en evolución. El examen patológico del pulmón muestra fibrosis con depósito de colágeno, inflamación crónica y aguda y resolución incompleta del edema. La fase de recuperación se caracteriza por resolución de la hipoxemia y mejora de espacio muerto y de la distensibilidad pulmonar. Las anormalidades radiológicas generalmente se resuelven, sin embargo, la fibrosis microscópica continúa. ${ }^{66}$ 


\title{
Interacción riñón-pulmón
}

\author{
Manuel Antonio Díaz de León-Ponce
}

\section{Introducción}

En soldados que murieron durante la Primera Guerra Mundial, los doctores Buford y Burbank encontraron edema en tejido pulmonar, que llamaron traumatic wet lung. En 1948, Moon describió en los pulmones de soldado muertos hiperemia, edema, hemorragia y atelectasias, cuya causa atribuyó a lesión endotelial. Dos años después, Jeakins reportó atelectasias y congestión pulmonar que consideró se debía a la sobrehidratación en la reanimación del paciente en estado de choque hipovolémico (Figura 7). $5,67,68$

Ashbaugh, a quien se conmemora en este simposio, hace cinco décadas describió el síndrome de insuficiencia respiratoria aguda (SIRA); precisó que su diagnóstico se confirma con datos clínicos, laboratorio y gabinete y lo clasificó en cuatro etapas (Figura 8). ${ }^{1}$

Siete años después, en México se publicó el primer estudio que confirmó los datos de Ashbaugh, pero se demostró que las etapas no dependían del tiempo sino de la intensidad de la lesión y de factores histológicos (Figura 9). ${ }^{69}$ Sin embargo, hace cinco décadas no se conocían los tres grandes síndromes que provocan la muerte del paciente crítico.

- Síndrome de respuesta inflamatoria sistémica (SRIS).

- Síndrome de respuesta inmune provocada por el SRIS.

- Síndrome de disfunción orgánica múltiple (SDOM). ${ }^{70-72}$

La mortalidad y morbilidad del paciente grave dependen de la severidad de SRIS, de la capacidad inmune del huésped y de la adaptabilidad y condiciones orgánicas del paciente. ${ }^{73}$

Al parecer, el efecto de la lesión renal y pulmonar no explica lo referido; desde hace cuatro décadas sabemos que esto depende de la respuesta inmune del enfermo, del SRIS y del daño de órganos como el corazón, bazo, cerebro, hígado e intestino, conjunción que se conoce como SDOM (Figura 10). En lo anterior intervienen la capacidad de cada ser humano ante la respuesta inflamatoria, la capacidad de cada órgano ante la lesión y la instauración de tratamiento temprano. ${ }^{68,73}$

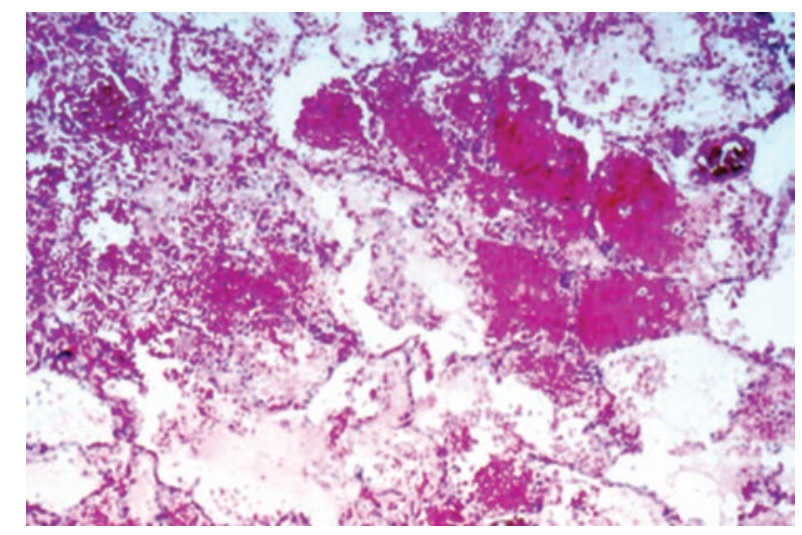

Figura 7. Imagen en la que se observa edema y hemorragia alveolar.
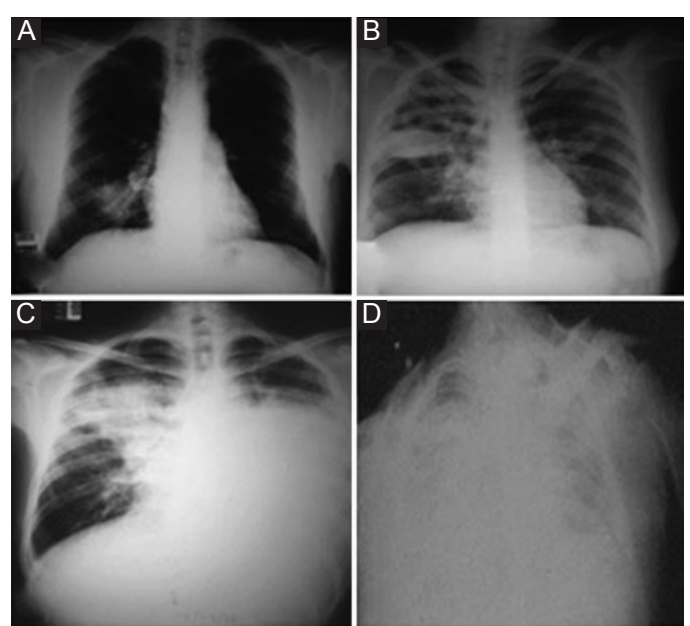

Figura 8. Etapas evolutivas del síndrome de insuficiencia respiratoria del adulto.

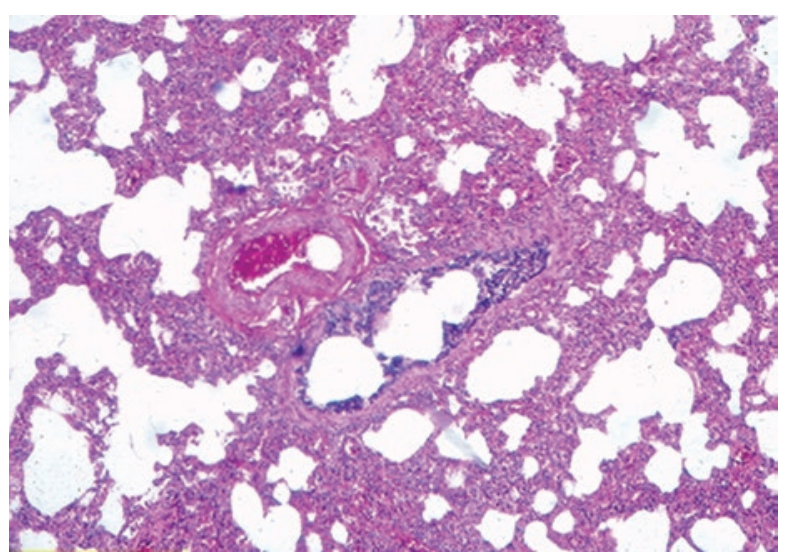

Figura 9. Biopsia pulmonar en la que se muestra daño alveolar difuso. 


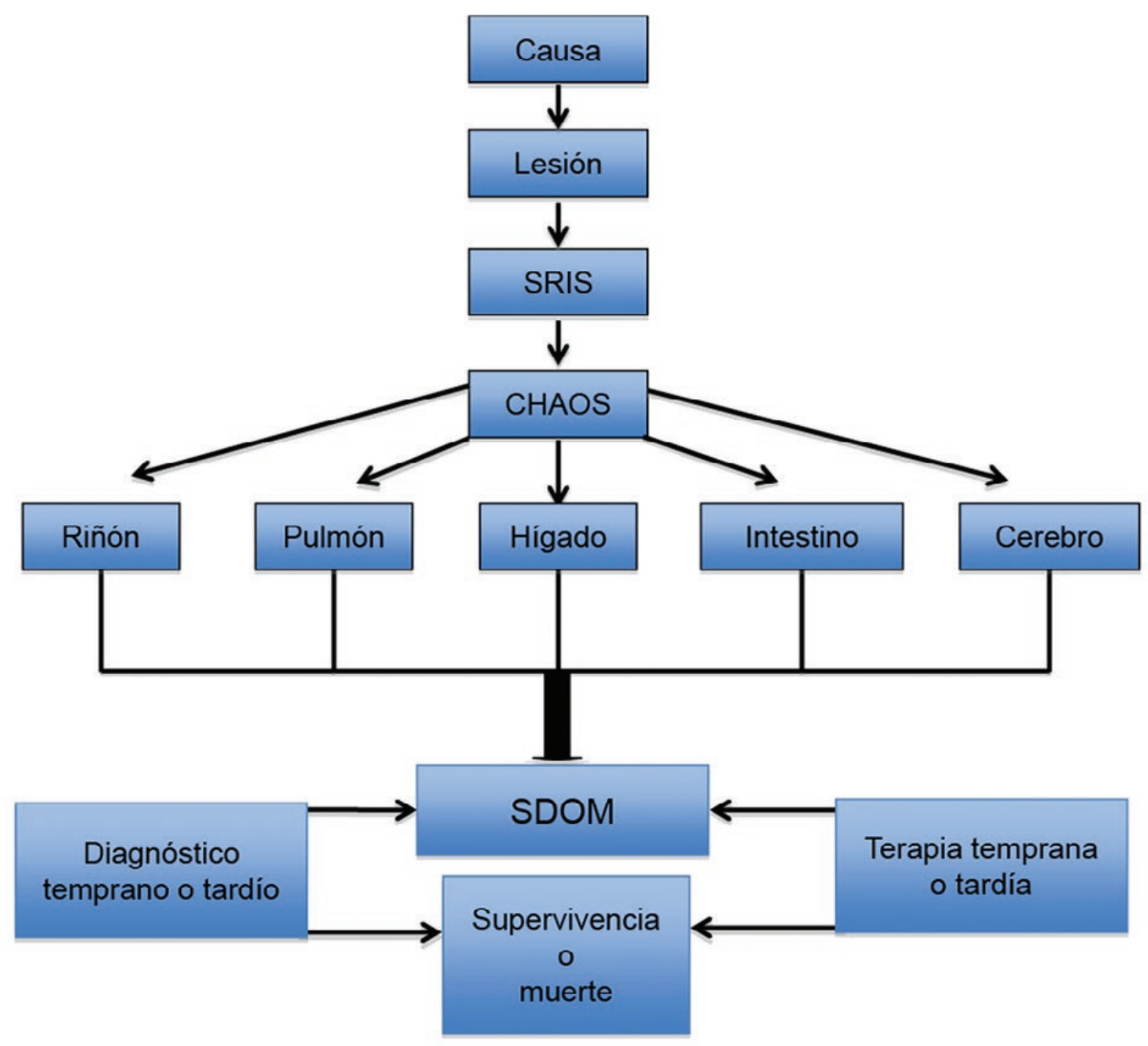

Figura 10. Fisiopatología de la disfunción multiorgánica. SRIS, síndrome de respuesta inflamatoria sistémica; SRCRS, síndrome de respuesta contrarreguladora sistémica; SDOM, síndrome de disfunción orgánica múltiple.

En 2009, Risso et al..$^{74}$ describieron que el síndrome pulmón-riñón se debía a hemorragia alveolar difusa y glomerulonefritis, lo relacionaron con vasculitis y colagenopatías e indicaron que era necesario iniciar un diagnóstico temprano y un tratamiento agresivo, con altas dosis de corticoides, inmunosupresores, inhibidores de necrosis tumoral y plasmaféresis (lo cual ya se había publicado en $1975^{75}$ ) (Figura 11), con el fin de evitar la acción de la respuesta inmunitaria de todos los anticuerpos propios de la enfermedad o de los diferentes mediadores de la inflamación. Estudios de 2011, 2012 y una revisión del grupo de Domenech et al. de 2017 tratan de confirmar lo anterior. ${ }^{76-79}$

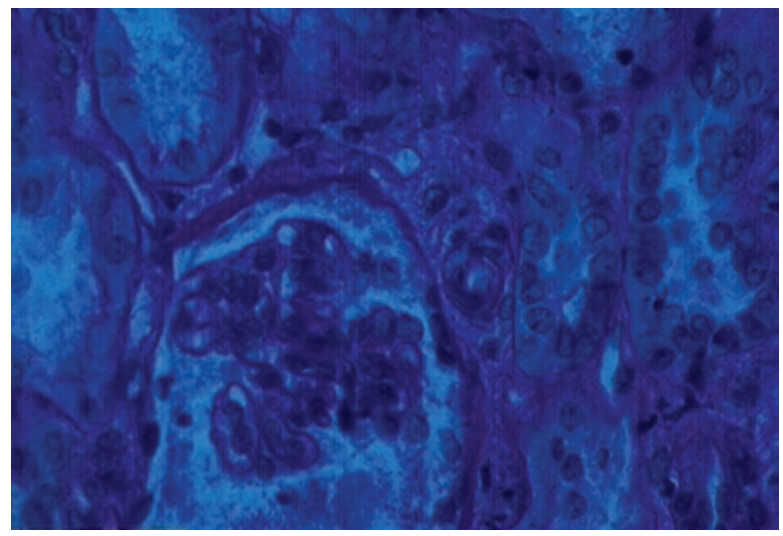

Figura 11. Biopsia renal de un enfermo con sepsis, en la que se observa imagen de glomerulonefritis. 


\section{HEMODIALISIS EXANGUINEOTRANSFUSION Y OXIGENACION}

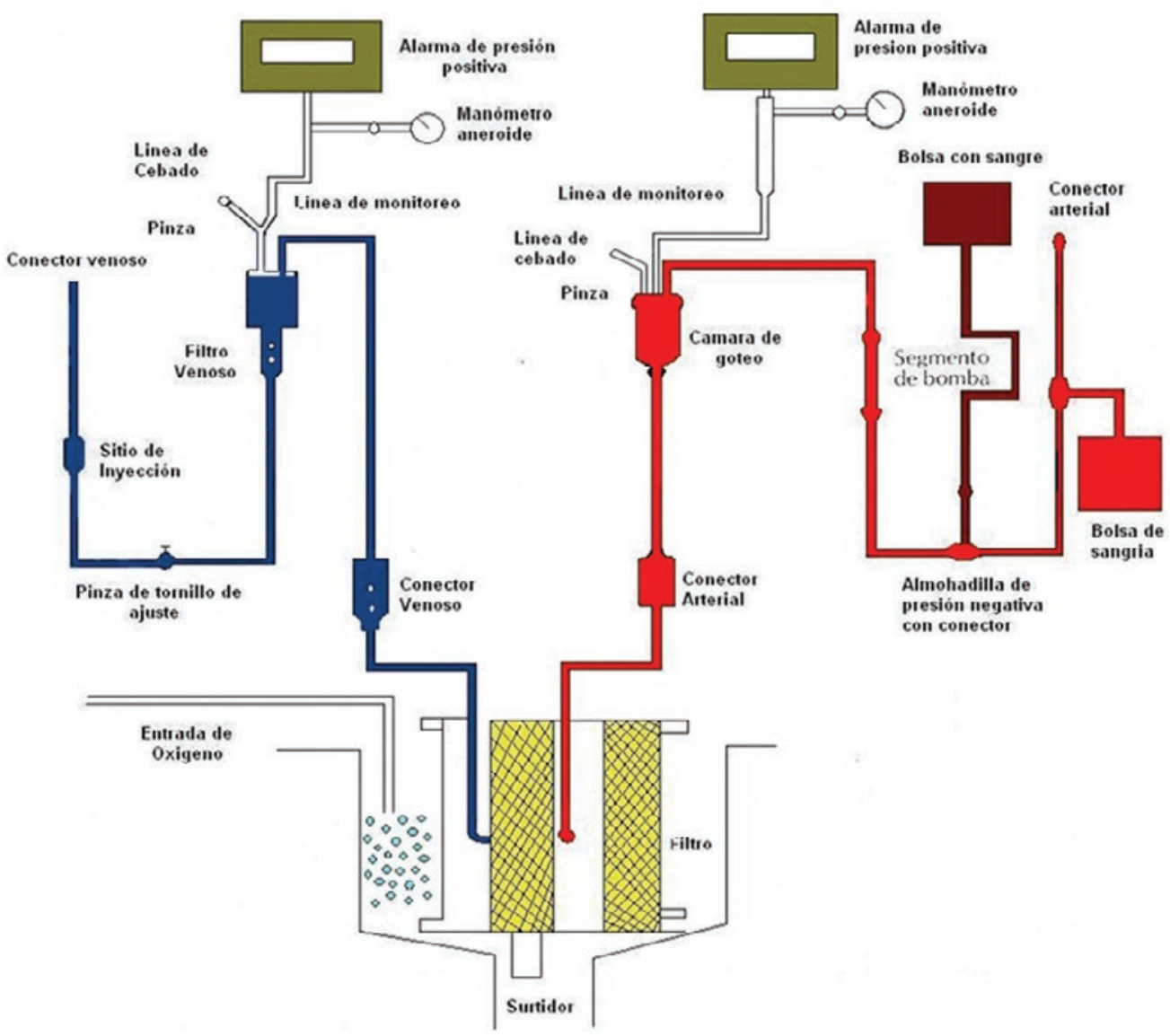

Figura 12. Diagrama en el que se muestra la técnica de hemodiálisis con oxigenación.

En 1980 publicamos como una novedad terapéutica el uso temprano de diálisis extracorpórea y exanguinotransfusión con barboteo de oxígeno, ${ }^{80}$ antecedentes de lo que mencionan Risso et al. (plasmaféresis y oxigenador de membrana) (Figura 12).

\section{Bibliografía}

1. Ashbaugh DG, Bigelow BD, Petty TL, Levine BE. Acute respiratory distress in adults. Lancet. 1967;2(7511):319-323.

2. Bellani G, Laffey JG, Pham T, Fan E, Brochard L, Esteban A, et al. Epidemiology, patterns of care, mortality for patients with acute respiratory distress syndrome in intensive care units in 50 countries. JAMA. 2016;315(8):788-800.

3. Villar J, Blanco J, Kacmarek RM. Current incidence and outcome of the respiratory distress syndrome. Curr Opin Crit Care. 2016;22(1):1-6.

4. Murray JF, Matthay MA, Luce JM, Flick MR. An expanded definition of the adult respiratory distress syndrome. Am Rev Respir Dis. 1988; 138(3):720-723.

5. Bernard GR, Artigas A, Brigham KL, Carlet J, Falke K, Hudson L, et al. The American-European Consensus Conference on ARDS. Definition, mechanisms, relevant outcomes, and clinical trials coordination. Am J Respir Crit Care Med. 1994;149(3 Pt 1):818-824.

6. ARDS Definition Task Force; Ranieri VM, Rubenfield GD, Thompson BT, Ferguson ND, Caldwell E, et al. Acute respiratory distress syndrome: The Berlin Definition. JAMA. 2012;307(23):2526-2533.

7. Han S, Mallampalli RK. The acute respiratory distress syndrome: from mechanism to translation. J Immunol. 2015;194(3):855-860.

8. Binnie A, Tsang JL, Dos-Santos CC. Biomarkers in acute respiratory distress syndrome. Curr Opin Crit Care. 2014;20(1):47-55.
9. Gattinoni L, Marini JJ, Pesenti A, Quientel M, Mancebo J, Brochard L. The "baby lung" became adult. Intensive Care Med. 2016; 42(5): 663-673.

10. Kallet RH. A comprehensive review of prone position in ARDS. Respir Care. 2015;60(11):1660-1687.

11. Brower RG, Matthay MA, Morris A, Schoenfeld D, Thompson BT, Wheeler $A$, et al. Ventilation with lower tidal volumes as compared with traditional tidal volumes for acute lung injury and the acute respiratory distress syndrome. N Engl J Med. 2000;342(18):1301-1308.

12. Malhorta A. Low-tidal-volume ventilation in the acute respiratory distress syndrome. N Engl J Med. 2007;357(11):1113-1120.

13. Matthay MA, Ware LB, Zimmerman GA. The acute respiratory distress syndrome. J Clin Invest. 2012;122(8):2731-2740.

14. Gonzales JN, Lucas R, Verin AD. The acute respiratory distress syndrome: mechanisms and perspective therapeutic approaches. Austin J Vasc Med. 2015;2(1):1009-1016.

15. Gattinoni L, Tonetti T, Cressoni M, Cadringher P, Hermann P, Moerer O, et al. Ventilator-related causes of lung injury: the mechanical power. Intensive Care Med. 2016:42(10):1567-1575.

16. Beitler JR, Malhorta A, Thompson BT. Ventilator-induced lung injury. Clin Chest Med. 2016;37(4):633-646.

17. Gattinoni L, Marini JJ, Collino F, Maiolo G, Rapetti F, Tonetti T, et al. The future of mechanical ventilation: lessons from de present and the past. Crit Care. 2017;21:183-194.

18. Guérin C, Reignier J, Richard JC, Beuret $P$, Gacounin A, Boulain T, et al. Prone positioning in severe acute respiratory distress syndrome. N Engl J Med. 2013;368(23):2159-2168.

19. Brodie D, Bacchetta M. Extracorporeal membrane oxygenation for ARDS in adults. N Engl J Med. 2011;365(20):1905-1914.

20. Rozé H, Repusseau B, Ouattara A. Extracorporeal membrane oxygenation in adults for severe acute respiratory failure. Ann Fr Anesth Reanim. 2014;33(7-8):492-494.

21. Amato MBP, Meade MO, Slutsky AS, Brochard L, Costa EL, Schoenfeld DA, et al. Driving pressure and survival in the acute respiratory distress syndrome. N Engl J Med. 2015;372:747-755. 
22. Nieman GF, Satalin J, Andrews P, Aiash H, Habashi NM, Gatto LA Personalizing mechanical ventilation according to physiologic parameters to stabilize alveoli and minimize ventilator induced lung injury (VILI). Intensive Care Med Exp. 2017;5:8-28.

23. Papazian L, Forel JM, Gacounin A, Penot-Ragon C, Perrin G, Loundou A, et al. Neuromuscular blockers in early acute respiratory distress syndrome. N Engl J Med. 2010;363(12):1107-1116.

24. Toner $P$, McAuley DF, Shyamsundar M. Aspirin as a potential treatment in sepsis or acute respiratory distress syndrome. Crit Care. 2015;19:374-380.

25. Rojas M, Xu J, Woods CR, Mora AL, Spears W, Roman J, et al. Bone marrow-derived mesenchymal stem cells in repair of the injured lung. Am J Respir Cell Mol Biol. 2005;33(2):145-152.

26. Duggal A, Ganapathy A, Ratnapalan M, Adhikari NK. Pharmacological treatments for acute respiratory distress syndrome: systematic review. Minerva Anestesiol. 2015;81(5):567-588.

27. Zhang Z, Chen L, Ni H. The effectiveness of corticosteroids on mortality in patients with acute respiratory distress syndrome or acute lung injury: a secondary analysis. Sci Rep. 2015:5:17654.

28. Attaway $\mathrm{AH}$, Myers $\mathrm{C}$, Velani S, Schilz R. Inhaled prostacyclin as a salvage therapy for ARDS: Can we find the right patient? Respir Care. 2017:62(8):1113-1115

29. Gattinoni L, Cressoni M, Brazzi L. Fluids in ARDS: from onset through recovery. Curr Opin Crit Care. 2014;20(4):373-377.

30. Valente-Barbas C, Janot-Matos GF, Passos-Amato M Ribeiro-Carvalho CR. Goal-oriented respiratory management for critically ill patients with acute respiratory distress syndrome. Crit Care Res Pract. 2012;2012:952-965

31. Herridge MS, Moss M, Hough CL, Hopkin RO, Rice TW, Bienvenue OJ, et al. Recovery and outcomes after the acute respiratory distress syndrome (ARDS) in patients and their family caregivers. Intensive Care Med. 2016:42(5):725-738.

32. Ware L, Matthay MA. The acute respiratory distress syndrome. N Engl J Med. 2000;342(18):1334-1349.

33. Máca J, Jor O, Holub M, Sklienka P, Burša F, Burda M, et al. Past and present ARDS mortality rates: a systematic review. Respir Care. 2017; 62(1):113-122.

34. Fan E, Del Sorbo L, Goligher EC, Hodgson CL, Munshi L, Walkey A, et al. An Official American Thoracic Society/European Society of Intensive Care Medicine/Society of Critical Care Medicine Clinical Practice Guideline: mechanical ventilation in adult patients with acute respiratory distress syndrome. Am J Respir Crit Care Med. 2017:195(9):1253-1263.

35. Rhodes A, Evans L, Alhazzani W, Levy MM, Antonelli M, Ferrer R, et al. Surviving sepsis campaign: International Guidelines for Management of Sepsis and Septic Shock: 2016. Crit Care Med. 2017;45(3):486-552.

36. Slutsky AS, Raniery VM. Ventilator-induced lung injury. N Engl J Med. 2014;369(22):2126-2136.

37. Meduri GU, Bridges L, Shih MC, Marik PE, Siemieniuk RA, Kocak M. Prolonged glucocorticoid treatment is associated with improved ARDS outcomes: analysis of individual patients' data from four randomized trials and trial-level meta-analysis of the updated literature. Intensive Care Med. 2016;42(5):829-840.

38. Adhikari NK, Dellinger RP, Lundin S, Payen D, Vallet B, Gerlach H, et al Inhaled nitric oxide does not reduce mortality in patients with acute respiratory distress syndrome regardless of severity: systematic review and meta-analysis. Crit Care Med. 2014;42(2): 404-412.

39. Sakr Y, Vincent JL, Reinhart K, Groeneveld J, Michalopoulos A, Sprung $C L$, et al. High tidal volume and positive fluid balance are associated with worse outcome in acute lung injury. Chest. 2005;128(5):3098-3108

40. Martin GS, Moss M, Wheeler AP, Mealer M, Morris JA, Bernard GR. A randomized, controlled trial of furosemide with or without albumin in hypoproteinemic patients with acute lung injury. Crit Care Med. 2005;33(8):1681-1687.

41. National Heart, Lung, and Blood Institute Acute Respiratory Distress Syndrome (ARDS) Clinical Trials Network; Wiedemann HP, Wheeler AP Bernard GR, Thompson BT, Hayden D, deBoisblanc B, et al. Comparison of two fluid-management strategies in acute lung injury. $\mathrm{N}$ Engl J Med. 2006;354(24):2564-2575.

42. Brochard L, Roudot-Thoraval F, Roupie E, Delclaux C, Chastre J, Fernández-Mondéjar $\mathrm{E}$, et al. Tidal volume reduction for prevention of ventilator-induced lung injury in acute respiratory distress syndrome. The Multicenter Trail Group on Tidal Volume reduction in ARDS. Am J Respir Crit Care Med. 1998:158(6):1831-1838.

43. Stewart TE, Meade MO, Cook DJ, Granton JT, Hodder RV, Lapinsky SE et al. Evaluation of a ventilation strategy to prevent barotrauma in patients at high risk for acute respiratory distress syndrome. N Engl J Med. 1998:338(6):355-361

44. Brower RG, Shanholtz CB, Fessler HE, Shade DM, White P, Wiener CM, et al. Prospective, randomized, controlled clinical trial comparing traditional versus reduced tidal volume ventilation in acute respiratory distress syndrome patients. Crit Care Med. 1999;27(8):1492-1498.
45. Passos-Amato MB, Valente-Barbas CS, Machado-Medeiros DM, Borges-Magaldi R, Schettino GP, Lorenzi-Filho G, et al. Effect of a protective-ventilation strategy on mortality in the acute respiratory distress syndrome. N Engl J Med. 1998;338:347-354.

46. The Acute Respiratory Distress Syndrome Network. Ventilation with lower tidal volumes as compared with traditional tidal volumes for acute lung injury and the acute respiratory distress syndrome. N Engl J Med. 2000;342:1301-1308.

47. Burns KE, Adhikari NK, Slutsky AS, Guyatt GH, Villar J, Zhang H, et al. Pressure and volume limited ventilation for the ventilatory management of patients with acute lung injury: a systematic review and meta-analysis. PLos One. 2011;6(1):e14623.

48. Lachmann B. Open up the lung and keep the lung open. Intensive Care Med. 1992;18(6):319-321.

49. Meade MO, Cook DJ, Guyatt GH, Slutsky AS, Arabi YM, Cooper DJ, et al. Ventilation strategy using low tidal volumes, recruitment maneuvers, and high positive end-expiratory pressure for acute lung injury and acute respiratory distress syndrome: a randomized controlled trial. JAMA. 2008:299(6):637-645.

50. Costa-Leme A, HajjarLA, Volpe MS, Fukushima JT, De-Santis-Santiago RR, Osawa $E$, et al. Effect of intensive vs moderate alveolar recruitment strategies added to lung-protective ventilation on postoperative pulmonary complications a randomized clinical trial. JAMA. 2017; 317(14):1422-1432.

51. Brower RG, Lanken PN, Macintyre N, Matthay MA, Morris A Ancukiewicz $M$, et al. Higher versus lower positive end-expiratory pressures in patients with the acute respiratory distress syndrome. N Engl J Med. 2004;351(4):327-336.

52. Mercat A, Richard JC, Vielle B, Jaber S, Osman D, Diehl JL, et al. Positive end-expiratory pressure setting in adults with acute lung injury and acute respiratory distress syndrome: a randomized controlled trial. JAMA. 2008;299(6):646-655.

53. Briel M, Meade M, Mercat A, Brower RG, Talmor D, Walter SD, et al. Higher vs lower positive end-expiratory pressure in patients with acute lung injury and acute respiratory distress syndrome: systematic review and meta-analysis. JAMA. 2010;303(9):865-873.

54. Guérin C, Papazian L, Reignier J, Ayzac L, Loundou A, Forel JM, et al. Effect of driving pressure on mortality in ARDS patients during lung protective mechanical ventilation in two randomized controlled trials. Critical Care. 2016;20:384

55. Ferguson ND, Cook DJ, Guyatt GH, Mehta S, Hand L, Austin P, et al. High-frequency oscillation in early acute respiratory distress syndrome. N Engl J Med. 2013;368(9):795-805.

56. Young D, Lamb SE, Shah S, MacKenzie I, Tunnicliffe W, Lall R, et al. High-frequency oscillation for acute respiratory distress syndrome. N Engl J Med 2013;368:806-813.

57. Chan KP, Stewart TE, Mehta S. High-frequency oscillatory ventilation for adult patients with ARDS. Chest. 2007;131(6):1907-1916.

58. Cooper DJ, Hodgson CL. Extracorporeal membrane oxygenation rescue for $\mathrm{H} 1 \mathrm{~N} 1$ acute respiratory distress syndrome: equipoise regained. Am J Respir Crit Care Med. 2013;187(3):224-226.

59. Peek GJ, Mugford M, Tiruvoipati R, Wilson A, Allen E, Thalanany MM, et al. Efficacy and economic assessment of conventional ventilatory support versus extracorporeal membrane oxygenation for severe adult respiratory failure (CESAR): a multicentre randomised controlled trial. Lancet. 2009;374(9698):1351-1363.

60. Terragni PP, Del-Sorbo L, Mascia L, Urbino R, Martin EL, Birocco A, et al. Tidal volume lower than $6 \mathrm{~mL} / \mathrm{kg}$ enhances lung protection: role of extracorporeal carbon dioxide removal. Anesthesiology. 2009; 111(4):8.

61. Bein T, Weber-Carstens S, Goldmann A, Müller T, Staudinger T, Brederlau J, et al. Lower tidal volume strategy ( $\approx 3 \mathrm{~mL} / \mathrm{kg}$ ) combined with extracorporeal $\mathrm{CO} 2$ removal versus 'conventional' protective ventilation $(6 \mathrm{~mL} / \mathrm{kg})$ in severe ARDS: the prospective randomized Xtravent-study. Intensive Care Med. 2013;39(5):847-856.

62. Fujishima S. Pathophysiology and biomarkers of acute respiratory distress syndrome. J Intensive Care. 2004;2(1):32.

63. Frantzeskaki F, Armaganidis A, Orfanos SE. Immunothrombosis in acute respiratory distress syndrome: cross talks between inflamation and coagulation. Respiration. 2017;93(3):212-225.

64. Yam-Puc JC, García-Marín LG, Sánchez-Torres LE. Trampas extracelulares de neutrófilos (NET), consecuencia de un suicidio celular. Gac Med Mex. 2012;148:68-75.

65. Díaz-Carrillo MA. Biología molecular de la ventilación mecánica. Rev Mex Anest. 2015;38(s1):s220-s222.

66. Díaz de León PM, Moreno SAA, González DJI, Briones GJC. Actualidades del síndrome de insuficiencia respiratoria aguda. Rev Asoc Mex Med Crit Ter Int. 2007;21(4):217-222.

67. Díaz de León-Ponce M, Mujica-Hernández M, Olvera-Chávez A, González-Díaz JI, Montealegre-Ramírez PA, Moreno-Santillán A, et al. Síndrome de insuficiencia respiratoria aguda (SIRA). Rev Asoc Mex Med Crit Ter Int. 2004;18(1):24-33. 
68. Díaz de León-Ponce M, Moreno-Santillán A, González-Díaz JI, Briones-Garduño JC. Actualidades del síndrome de insuficiencia respiratoria aguda. Rev Asoc Mex Med Crit Ter Int. 2007;21(4):217-222.

69. Díaz de León-Ponce M, Terán EH, Ramírez OJ. Pulmón de choque en gineco-obstetricia. Ginec Obstet Mex. 1975;37:197-206.

70. Tilney NL, Barley GL, Morgan AP. Sequential system failure after rupture of abdominal aortic aneurysms. An unsolved problem in postoperative care. Ann Surg. 1973;178(2):117-122.

71. Rangel-Frausto MS, Pittet D, Costigan M, Hwang T, Davis CS, Wenzel RP. The natural history of the systemic inflammatory response syndrome. A prospective study. JAMA. 1995;273(2):117-123.

72. Feregrino GM, Díaz de León-Ponce M. Inmunología en el estado crítico. En: Díaz de León-Ponce M. Medicina crítica. México: Prado; 1990.

73. Carrillo ER. Sepsis. México: Alfil; 2009.

74. Risso JA, Mazzocchi O, De-All J, Gnocchi CA. Síndrome pulmón-riñón. Medicina (B. Aires). 2009;69(6):663-673.
75. Díaz de León-Ponce $\mathrm{M}$, Exaire $\mathrm{ME}$, Pizzuto $\mathrm{CH}$. Glomerulonefritis rápidamente progresiva tratada con anticoagulantes y antitrombóticos. Bol Med IMSS. 1975;1:49-54.

76. Doi K, Fujita T, Noiri E, Ishizu T. Lung injury following acute kidney injury: kidney-lung crosstalk. Clin Exp Nephol. 2011;15(4):464-470.

77. Izaguirre A, Herrando S, Daborro M, Bauni C. Síndrome pulmón-riñón. Rev Hosp Ital B Aires. 2012;32(3):1-3.

78. Faubel $\mathrm{S}$, Edelstein $\mathrm{CL}$. Mechanisms and mediators of lung injury after acute kidney injury. Nat Rev Nephrol. 2016;12(1):48-60.

79. Domenech P, Pérez T, Saldarini A, Uad P, Musso CG. Kidney-lung pathophysiological crosstalk: its characteristics and importance. Int Urol Nephrol. 2017;49(7):1211-1215.

80. Díaz de León-Ponce M, Foubert DC, Díaz MT, López FP, Ramírez HL, Martínez GM. Hemodiálisis-exanguinotransfusión y oxigenación en choque séptico. Un nuevo procedimiento. Nefrologia Mex. 1980; 1:37-42. 